\title{
Abnormal Changes of Multidimensional Surface Features Using Multivariate Pattern Classification in Amnestic Mild Cognitive Impairment Patients
}

\author{
Shuyu Li, ${ }^{1}$ Xiankun Yuan, ${ }^{1}$ Fang Pu, ${ }^{1}$ Deyu Li, ${ }^{1}$ Yubo Fan, ${ }^{1}$ Liyong Wu, $, 2,3$ Wang Chao, ${ }^{4}$ Nan Chen, ${ }^{4}$ Yong He, ${ }^{5}$ \\ and Ying $\operatorname{Han}^{2,3}$ \\ ${ }^{1}$ School of Biological Science \& Medical Engineering, Beihang University, Beijing 100191, China, ${ }^{2}$ Department of Neurology, Xuanwu Hospital, Capital \\ Medical University, Beijing 100053, China, ${ }^{3}$ Center of Alzheimer's Disease, Beijing Institute for Brain Disorders, Beijing 100053, China, ${ }^{4}$ Department of \\ Radiology, Xuanwu Hospital, Capital Medical University, Beijing 100053, China, and ${ }^{5}$ State Key Laboratory of Cognitive Neuroscience and Learning \& IDG/ \\ McGovern Institute for Brain Research, Beijing Normal University, Beijing 100875, China
}

Previous studies have suggested that amnestic mild cognitive impairment (aMCI) is associated with changes in cortical morphological features, such as cortical thickness, sulcal depth, surface area, gray matter volume, metric distortion, and mean curvature. These features have been proven to have specific neuropathological and genetic underpinnings. However, most studies primarily focused on massunivariate methods, and cortical features were generally explored in isolation. Here, we used a multivariate method to characterize the complex and subtle structural changing pattern of cortical anatomy in 24 aMCI human participants and 26 normal human controls. Six cortical features were extracted for each participant, and the spatial patterns of brain abnormities in aMCI were identified by high classification weights using a support vector machine method. The classification accuracy in discriminating the two groups was $76 \%$ in the left hemisphere and $80 \%$ in the right hemisphere when all six cortical features were used. Regions showing high weights were subtle, spatially complex, and predominately located in the left medial temporal lobe and the supramarginal and right inferior parietal lobes. In addition, we also found that the six morphological features had different contributions in discriminating the two groups even for the same region. Our results indicated that the neuroanatomical patterns that discriminated individuals with aMCI from controls were truly multidimensional and had different effects on the morphological features. Furthermore, the regions identified by our method could potentially be useful for clinical diagnosis.

Key words: aMCI; cortical surface feature; entorhinal; MRI; multivariate classification

\section{Introduction}

Mild cognitive impairment (MCI) represents the transition state between normal age-related cognitive changes and Alzheimer's disease (AD; Petersen, 2003). Amnestic MCI (aMCI) refers to one MCI subtype characterized by primary memory deficits and has a high risk of progression to AD (Petersen et al., 2001a, b). Previous MRI studies have reported that aMCI subjects have gray matter (GM) atrophy or volume decline in the entorhinal cortex, the

Received Oct. 9, 2013; revised May 2, 2014; accepted June 2, 2014.

Author contributions: S.L., Y.He, and Y.Han designed research; S.L., X.Y., W.C., N.C., and Y. Han performed research; F.P., D.L., and Y.F. contributed unpublished reagents/analytic tools; S.L., X.Y., F.P., and D.L. analyzed data; S.L., X.Y., Y.F., L.W., Y. He, and Y. Han wrote the paper.

This work was supported by the National Science Foundation of China $(81171403,30970823,31371007$, and 81030028), the Beijing Municipal Science \& Technology Commission (Grant Z131100006813022), the National Science Fund for Distinguished Young Scholars (81225012), and the National Key Department of Neurology funded by the Chinese Health and Family Planning Committee.

The authors declare no competing financial interests.

Correspondence should be addressed to either of the following: Dr. Shuyu Li, School of Biological Science \& Medical Engineering, Beihang University, Beijing 100191, China, E-mail: shuyuli@buaa.edu.cn; or Dr. Ying Han, Department of Neurology, Xuanwu Hospital, Capital Medical University, Beijing 100053, China, E-mail: 13621011941@163.com.

DOI:10.1523/JNEUROSCI.4356-13.2014

Copyright $\odot 2014$ the authors $\quad 0270-6474 / 14 / 3410541-13 \$ 15.00 / 0$ posterior cingulate, and the medial prefrontal cortex (Apostolova et al., 2007; Seo et al., 2007).

Morphological parameters, based on a 3D surface model of the cerebral cortex, have been widely used to detect the brain abnormalities of aMCI (Lerch et al., 2005a; Apostolova et al., 2007; Frisoni et al., 2007). These parameters that measure volumetric and geometric features of the cerebral cortex, such as cortical thickness, surface areas, GM volume, sulcal depth, metric distortion, and mean curvature, have been proven to have unique neuropathological and genetic underpinnings (Rakic., 1988; Huttenlocher, 1990; Van Essen, 1997; Panizzon et al., 2009). The definitions and physiological meanings of the various morphological measures are shown in Table 1. Several studies have reported abnormalities in these morphological parameters in aMCI subjects. For example, aMCI patients showed overall cortical thinning and sulcal widening compared with normal controls (Liu et al., 2013) and a reduced average mean curvature in the temporal lobe (Im et al., 2008). Compared with healthy elderly, aMCI patients lost asymmetry in the entorhinal cortex as measured by cortical thickness (Long et al., 2013). However, these studies used a mass-univariate method, and the cortical features were generally explored in isolation. 
Table 1. Definitions and meanings of the six morphological measures

\begin{tabular}{ll}
\hline Morphological measures & Definitions \\
$\begin{array}{l}\text { Cortical thickness } \\
\text { Surface area }\end{array}$ & $\begin{array}{l}\text { The closest distance between the white and pial surfaces at each vertex on the cortical surface } \\
\text { The average area of the triangles that touch the vertex on the pial surface }\end{array}$ \\
$\begin{array}{l}\text { GM volume } \\
\text { Sulcal depth }\end{array}$ & $\begin{array}{l}\text { The sum of the volumes of the individual triangles that lie within the neighborhood of the vertex } \\
\text { Metric distortion }\end{array}$ \\
$\begin{array}{ll}\text { Mean curvature } \\
\text { The degree of displacement and convolution of the cortical surface relative to the average template }\end{array}$ \\
\hline
\end{tabular}

The multivariate method, which is superior to the massunivariate method, treats all cortical features together and allows us to determine the relationships among different features beyond their individual values (Johnson, 2005). Thus, this approach could provide valuable insights into the multifactorial etiology of neurologic disease. The multivariate method has previously been applied in some brain disorders, such as autism spectrum disorder (Ecker et al., 2010), multiple sclerosis (Bendfeldt et al., 2012), and schizophrenia (Yu et al., 2013). For example, Ecker et al. (2010) found that different cortical features in the inferior parietal lobe and the temporal sulcus had unequal contributions in discriminating individuals with autism spectrum disorders from normal controls by using the multivariate method. Additionally, Westman et al. (2013) found that a high classification accuracy could be obtained by using un-normalized cortical thickness and normalized volumes and a multivariate analysis in $\mathrm{AD}$ and MCI patients. However, there are few studies that investigated abnormalities of multiple cortical features in aMCI patients using the multivariate method.

Here, we used a multivariate computational approach that combined six surface features to investigate the cortical changes between aMCI and normal control (NC) groups. Due to abnormal changes in multiple cortical features in aMCI participants revealed by previous studies, we expected to observe multidimensional neuroanatomical patterns in discriminating the aMCI patients from the controls. Furthermore, we explored whether these features have equal contributions when differentiating the two groups.

\section{Materials and Methods}

Participants. Twenty-four aMCI subjects ( 6 males and 18 females) and 26 demographically matched controls (11 males and 15 females) were recruited through a clinical research program at the Xuan Wu Hospital/ Institute of Neurology (Beijing, China). This study was approved by the Research Ethics Review Board of Xuan Wu Hospital, and all volunteers provided informed consent. All subjects were right-handed and were between 54 and 68 years of age. The diagnosis of aMCI was determined by the consensus of two experienced neurologists according to the criteria for aMCI (Petersen et al., 1999, 2001a,b), which included: (1) memory complaint, preferably confirmed by an informant; (2) objective memory impairment, adjusted for age and education; (3) normal or near-normal performance on general cognitive functioning and no or minimal impairment of daily life activities; (4) a Clinical Dementia Rating (CDR) score of 0.5 ; and (5) not meeting the criteria for dementia according to the Diagnostic and Statistical Manual of Mental Disorders, fourth edition, revised (DSM-IV). The healthy controls were screened using the Structured Interview for DSM-IV Non-Patient Edition to confirm the lifetime absence of psychiatric and neurological illnesses. The following exclusion criteria applied to all subjects: the existence of a neurological disorder, alcohol or drug abuse, or any physical illness, such as hepatitis, brain tumor, trauma, or epilepsy, as assessed based on clinical evaluations and medical records. The demographic and clinical data of the participants are presented in Table 2.
Table 2. Subject demographics

\begin{tabular}{lccr}
\hline & $\mathrm{aMCl}(n=24)$ & Control $(n=26)$ & $p$ value \\
\hline Gender (M/F) & $6 / 18$ & $11 / 15$ & 0.206 \\
Age & $61.0 \pm 4.3(54-68)$ & $60.0 \pm 1.4(57-63)$ & 0.179 \\
Education & $8.7 \pm 4.6(0-21)$ & $9.3 \pm 3.4(0-15)$ & 0.581 \\
MMSE & $24.3 \pm 3.6(17-30)$ & $28.3 \pm 2.1(20-30)$ & $<0.001$ \\
MoCA & $19.7 \pm 3.8(15-30)$ & $26.5 \pm 3.1(18-30)$ & $<0.001$ \\
\hline
\end{tabular}

Age, education, MMSE, and MoCA data are expressed as the mean \pm SD (range). There were no significant differences between the two groups in gender, age, or education years. The aMCI and NC groups showed significant differences in the MMSE and MoCA scores $(p<0.01)$. Statistical $p$ value was analyzed using a $t$ test, except for gender (Wilcoxon test).

MRI data acquisition. MRI data were acquired on a 3.0 T Siemens scanner by using a sagittal MP-RAGE sequence with the following imaging parameters: $\mathrm{TR}=1900 \mathrm{~ms}$; $\mathrm{TE}=2.2 \mathrm{~ms}$; inversion time $=900 \mathrm{~ms}$; flip angle $=9$ degrees; FOV $=250 \mathrm{~mm} \times 250 \mathrm{~mm}$; matrix $=256 \times 256$; 176 slices, thickness $=1.0 \mathrm{~mm}$. Brain MR images were inspected by an experienced neuroradiologist, and no gross abnormality was observed for any subject.

Image processing. Cortical reconstruction and morphological feature extraction were performed using the FreeSurfer image analysis suite (http://surfer.nmr.mgh.harvard.edu/), with a standard cortical automatic handling protocol. Briefly, the MRI data were first normalized to a standard anatomical template (Talairach and Tournoux, 1988) and corrected for bias-field inhomogeneities. Then, the resulting images were skull stripped using a watershed algorithm (Ségonne et al., 2004) and subsequently segmented into the subcortical white matter and deep GM volumetric structures (Fischl et al., 2002, 2004b). The initial tessellation was formed by reconstructing the GM/white matter boundary (white surface) and the outer cortical surface (pial surface; Dale et al., 1999; Fischl et al., 2000). Subsequently, a series of deformation procedures was performed, including surface inflation (Dale et al., 1999), registration to a spherical atlas (Fischl et al., 1999), and parcellation of the cerebral cortex into units based on the gyral and sulcal structures (Fischl et al., 2004b). All reconstructed surfaces were visually inspected for gross anatomical topological defects. Finally, a variety of morphological features at each vertex on the pial surface were computed, including volumetric (cortical thickness, surface area, and GM volume) and geometric (sulcal depth, metric distortion, and mean curvature) measures. It is worth mentioning that the thickness across the cortical mantle was extracted by computing the closest distance between the white and pial surfaces at each vertex on the tessellated surface (Fischl et al., 2000). The surface area at each vertex was calculated as the average of the area of the triangles touching that vertex on the pial surface. The GM volume measure at each vertex was defined as the sum of the volumes of the individual triangles that lie within the neighborhood of the vertex, where the volume of each triangle was computed as the product of its area and the thickness at the center of the triangle. Sulcal depth measures the displacement from each vertex to the average surface. The average surface is a hypothetical "midsurface" that exists between the gyri and sulci. The metric distortion (i.e., Jacobian), which indicated the degree of cortical folding, was calculated as the degree of displacement and convolution of the cortical surface relative to the average template (Fischl et al., 1999; Wisco et al., 2007). The mean (radial) curvature was measured as $1 / r$ ( $r$ is the radius of an inscribed circle) and was used to assess the folding of the small secondary 
and tertiary folds in the surface. All surface features were smoothed using a $20 \mathrm{~mm}$ FWHM surface-based diffusion kernel to improve the ability to detect groupwise differences. For these smoothed parameters, we removed the effects of age using a general linear model in FreeSurfer and applied them in the further multivariate analysis. Additional technical details of these procedures have been described in previous publications (Dale et al., 1999; Fischl et al., 1999, 2000, 2002, 2004a,b; Ségonne et al., 2004; Jovicich et al., 2006).

Classification using support vector machine. A linear support vector machine (SVM) was used to classify the differences between the two groups on the basis of their cortical morphological features. An SVM is a supervised multivariate classification method that identifies the optimal hyperplane with the maximum margin (i.e., maximal separation between classes; Vapnik, 1999). A large body of studies has applied an SVM in MRI data classification (Burges, 1998; Schoelkopf et al., 2002; MourãoMiranda et al., 2006; Davatzikos et al., 2008; Klöppel et al., 2008). Our procedures were as follows. First, all features extracted from each image constructed a feature vector. The number of dimensions of each feature vector equaled the number of voxels/vertices per image multiplied by the number of measures. Thus, each image was represented by a feature vector and treated as a point in a high-dimensional space. Then, an SVM was used to train on a subset of the data $\langle x, c\rangle$ to identify a hyperplane that best separated the input space according to the class labels $c$ (e.g., +1 for patients, -1 for controls), where $x$ represented the input data (i.e., feature vector). The hyperplane was defined by a weight vector, which was a linear combination of the support vectors and an offset. In this study, a linear kernel function was used to train the SVM classifier because this ensured the weight vector could be represented as an image (i.e., the SVM discrimination map). The penalty parameter $C$ of the error term was fixed at $C=1$ for all cases (default value), which controlled the trade-off between having zero training errors and allowing misclassifications. For each hemisphere, we trained the respective classifier. Finally, the different SVM classifiers were trained using each individual morphological feature and a combination of all features. The LIBSVM toolbox for MATLAB was used to perform the classifications (http://www.csie.ntu.edu.tw/ cjlin/libsvm/).

Discrimination maps. The discrimination map was an image constructed by the weight vector that was acquired after the SVM training. The weight vector was normal to the hyperplane and had equal dimensions with the feature vector. Its value was related to the contribution of discriminating two classes. In this study, each feature vector had $n \times m$ dimensions, where $n$ equaled the number of vertices (here $n=163,842$ ) and $m$ denoted the number of measures (i.e., six). Due to the two groups (aMCI vs controls) with the labels +1 and -1 , respectively, a positive weight value in the discrimination map (red/yellow color scale) indicated relatively higher feature values in the aMCI patients compared with the controls with respect to the hyperplane and vice versa (blue/cyan color scale). To enable visualization of the discriminating pattern for each morphological measure, the weight vector was cut into its constituent parts and then mapped back onto the average white matter surface. We visualized all brain regions with color bars that represented their contributed weights.

Intraregional morphometric profiles. To identify the different contribution of each morphological measure in discriminating the two groups on the cortical surface, we displayed the intraregional morphometric profiles. These profiles were derived by calculating the average weights of the vertices within the ROIs for the six different parameters. The choice of the ROI was motivated by the high-parameter weights for a specific morphometric feature (e.g., high weights for the cortical thickness in the parahippocampal gyrus). The ROIs were defined as contiguous weight clusters from the overall discrimination maps. The ROI analysis aimed to illustrate that different cortical features had different contributions in the classification of aMCI and NC for a specific region, and thus might be related to different pathological causes of aMCI.

Cross-validation and permutation testing. The performance of the classifier was validated using a leave-one-out cross validation approach. In each leave-one-out cycle, one subject was removed from the whole group (aMCI and NC) and used as the test sample. The remaining subjects were used to train the classifier, and the constructed classifier was then used to
Table 3. Between-group differences in the intracranial volume, overall brain volume, and $\mathrm{GM}$ volume

\begin{tabular}{llrll}
\hline & \multicolumn{1}{l}{$\begin{array}{l}\text { aMCl } \\
\left(\times 10^{5} \mathrm{~mm}^{3}\right)\end{array}$} & \multicolumn{1}{c}{$\begin{array}{l}\text { Control } \\
\left(\times 10^{5} \mathrm{~mm}^{3}\right)\end{array}$} & \multicolumn{1}{c}{$t_{(48)}$} & \multicolumn{1}{l}{$P$} \\
\hline ICV & $13.40 \pm 0.15$ & $1.39 \pm 0.14$ & -1.14 & 0.260 \\
Total brain volume & $9.67 \pm 0.79$ & $10.30 \pm 1.15$ & -2.35 & 0.013 \\
Total GM volume & $4.26 \pm 0.35$ & $4.57 \pm 0.44$ & -2.71 & 0.007 \\
Right GM volume & $2.12 \pm 0.16$ & $2.28 \pm 0.22$ & -2.75 & 0.008 \\
Left GM volume & $2.14 \pm 0.18$ & $2.29 \pm 0.22$ & -2.66 & 0.011 \\
\hline
\end{tabular}

Data are expressed as the means $\pm S D$. ICV, total intracranial volume.

Table 4. Results of the SVM classification between the aMCl and control groups using different brain morphometric features in the left and right hemispheres

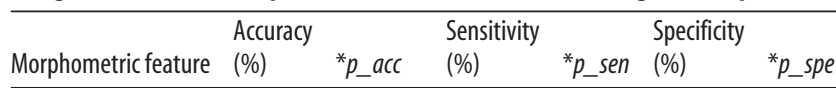

\begin{tabular}{lrrlrlr}
\hline Left hemisphere & & & & & & \\
All parameters & 76 & 0.0126 & 58 & 0.2478 & 92 & 0.0040 \\
Cortical thickness & 78 & 0.0020 & 62 & 0.1470 & 92 & $<0.0002$ \\
Sulcal depth & 58 & 0.1774 & 50 & 0.4338 & 65 & 0.1008 \\
Surface area & 66 & 0.0484 & 62 & 0.1518 & 69 & 0.0704 \\
Metric distortion & 84 & $<0.0002$ & 75 & 0.0148 & 92 & $<0.0002$ \\
Mean curvature & 76 & 0.0094 & 54 & 0.3248 & 96 & 0.0004 \\
GM volume & 64 & 0.0604 & 54 & 0.3246 & 73 & 0.0348 \\
Right hemisphere & & & & & & \\
All parameters & 80 & 0.0040 & 66 & 0.1080 & 92 & 0.0040 \\
Cortical thickness & 60 & 0.1326 & 45 & 0.5876 & 73 & 0.0334 \\
Sulcal depth & 78 & 0.0020 & 66 & 0.0790 & 88 & 0.0006 \\
Surface area & 76 & 0.0044 & 66 & 0.0930 & 85 & 0.0030 \\
Metric distortion & 60 & 0.1148 & 54 & 0.3300 & 65 & 0.0850 \\
Mean curvature & 62 & 0.0988 & 54 & 0.3142 & 69 & 0.0696 \\
GM volume & 56 & 0.2186 & 41 & 0.7072 & 69 & 0.0642 \\
\hline
\end{tabular}

${ }^{*} p_{\text {_ a acc }}\left({ }^{*} p_{\text {_ s sen, }}{ }^{*} p_{\text {_ }}\right.$ spec) values indicate the percentage that the accuracies (sensitivities, specificities) for the permuted labels were higher than the ones obtained for the real labels in 5000 permutations tests.

test the subject who had been removed. This procedure was repeated 50 times, i.e., the total sample size of the group. The classification accuracy was measured by the proportion of observations that were correctly classified into the patient or control groups. In addition, we evaluated the sensitivity and specificity of the classifier, where sensitivity was defined as $T P /(T P+F N)$, and specificity was defined as $T N /(T N+F P)$. The $T P$ (i.e., true positive) is the number of patient images correctly classified, whereas the $T N$ (i.e., true negative) is the number of control images correctly classified. The FP (i.e., false positive) is the number of controls images classified as patients, whereas the $F N$ (i.e., false negative) is the number of patient images classified as controls. Subsequently, the basic receiver operating characteristic (ROC) graphs were plotted. The classifications were performed by including all six morphological measures into the feature vector, as well as each individual measure.

The classifier performance was further evaluated using permutation testing, which computed the probability of getting specificity and sensitivity values higher than the ones obtained during the cross-validation procedure by chance. We randomly assigned patient and control labels to each subject and performed the cross-validation procedure. This process was repeated 5000 times. We then counted the percentage that the accuracies for the permuted labels were higher than the ones obtained for the real labels as the $p$ value.

\section{Results}

\section{Between-group volumetric differences}

Group differences in the intracranial volume, the total brain volume, and the GM volume were assessed using two-sample $t$ tests before classification. There was no significant between-group difference in the intracranial volume at a level of $p<0.05$ (Table 3), but significant differences were found in the total brain volume, the total GM volume, and the bilateral GM volumes between the two groups at $p<0.05$. 


\section{Overall classifier performance}

The classification accuracies, as well as the sensitivity and specificity for each classifier, are listed in Table 4. For the left hemisphere, high accuracies were observed in all features, including the cortical thickness, surface area, sulcal depth, metric distortion, mean curve, and GM volume. The permutation test $p$ values for these classifiers were $<0.05$. Thus, the probability of obtaining accuracies higher than the ones obtained during the cross-validation procedure by chance is extremely low. If a classifier has high sensitivity and specificity, it corresponds to a point in the upper left part of the ROC graph. In the left hemisphere, the best discrimination was obtained when the metric distortion measures were used to classify the difference between the groups with $75 \%$ sensitivity and $92 \%$ specificity (Fig. $1 A$ ). The individuals were correctly assigned to the appropriate diagnostic category in $76 \%$ of cases when all parameters were considered simultaneously (Fig. 1C). The specificity of the multiparameter classification in the left hemisphere was $92 \%$, which indicated that $92 \%$ of the controls subjects were correctly classified as controls.

For the right hemisphere, we obtained a similar parameter importance profile. High accuracies were observed in all features, including the sulcal depth and the surface area. The permutation test $p$ values for these classifiers were $<0.05$. In the ROC graph, the best discrimination was observed when all measures were used to classify between groups with $66 \%$ sensitivity and $92 \%$ specificity (Fig. $1 B$ ). The individuals were correctly assigned to the appropriate diagnostic category in $80 \%$ of cases when all parameters were considered simultaneously (Fig. 1D).

To test the effectiveness of the classifier, we computed the correlation coefficients between the Mini Mental State Examination (MMSE), the Montreal Cognitive Assessment (MoCA), and the test margin (the decision value of the weight vector) obtained from the combined model. The results are listed in Table 5. The test margin in both hemispheres was negatively correlated with the MMSE (Left: $r=-0.5198, p=0.0001$; Right: $r=-0.4656$, $p=0.0007$ ) and the MoCA scores (Left: $r=-0.5655, p<0.0001$; Right: $r=-0.4925, p=0.0003)$. Therefore, the individuals with higher values on the MMSE or the MoCA scores were located on the extreme left relative to the hyperplane, while the individuals with a lower level of these diagnostic criteria scores were predominately located right to the hyperplane overall.

Effects of different combinations of features on classification To test the effects of different combinations with subsets of the six morphological parameters on the classification performance, we chose the combinations of any two features to construct the classifier. The scatter diagrams of the classification results are shown in Figure 2. Our experiments showed that the different feature combinations yielded different classification results. For example, for the left hemisphere (Fig. 2A), the highest classification accuracy was obtained by the combination of metric distortion and cortical thickness
Table 5. Correlation coefficients between the aMCI diagnostic criteria MMSE, the $\mathrm{MoCA}$, and the weight vector for the model that combined all parameters

\begin{tabular}{llllll}
\hline & \multicolumn{2}{l}{ Left hemisphere } & & \multicolumn{2}{l}{ Right hemisphere } \\
\cline { 2 - 3 } Diagnostic test & $r$ & & $r$ & $p$ \\
\hline MMSE $(n=50)$ & -0.5198 & 0.0001 & & -0.4656 & 0.0007 \\
MoCA $(n=50)$ & -0.5655 & $<0.0001$ & & -0.4925 & 0.0003 \\
\hline
\end{tabular}

features. For the right hemisphere (Fig. $2 B$ ), the combination of all features obtained the highest classification accuracy, which was higher compared with 15 pairwise combinations.

In addition, we also constructed the classifiers by combining the left and right hemispheres and compared the results with the individual hemispheres (Fig. 2C). Our results showed that the classification performances after including the two hemispheres were not higher compared with those derived from the individual hemispheres except for the sulcal depth measurement.

We also added the MMSE and the MoCA in the SVM classification for each hemisphere. Here, we performed the permutation tests 5000 times and gave the null histograms for the different conditions, such as the pure morphometric-based classification, the morphometric feature with the MMSE and MoCA, the MMSE alone, the MoCA alone, and the MMSE and MoCA combined classification. The detailed results are shown in Figure 3 (left hemisphere), Figure 4 (right hemisphere), and Figure 5 (MMSE and MoCA). In these experiments, we did not observe obvious changes in the classification results by comparing the pure morphometric-based classification to the morphometric 
A

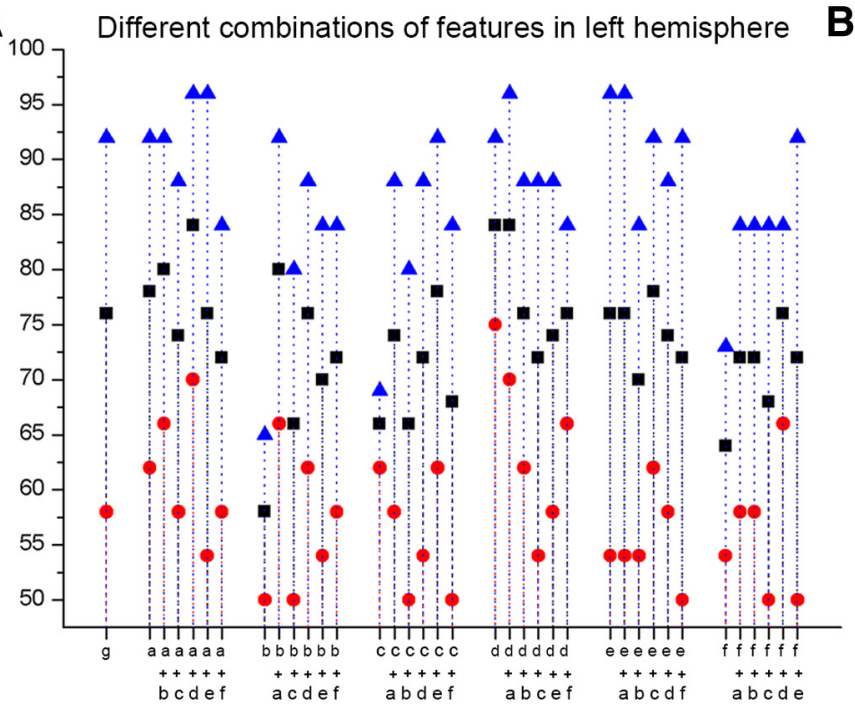

B

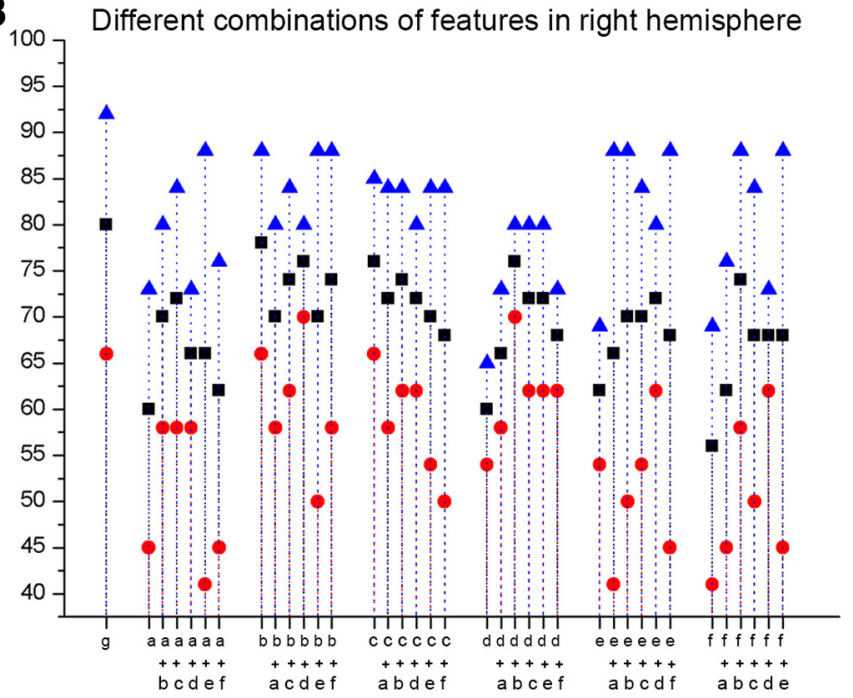

C

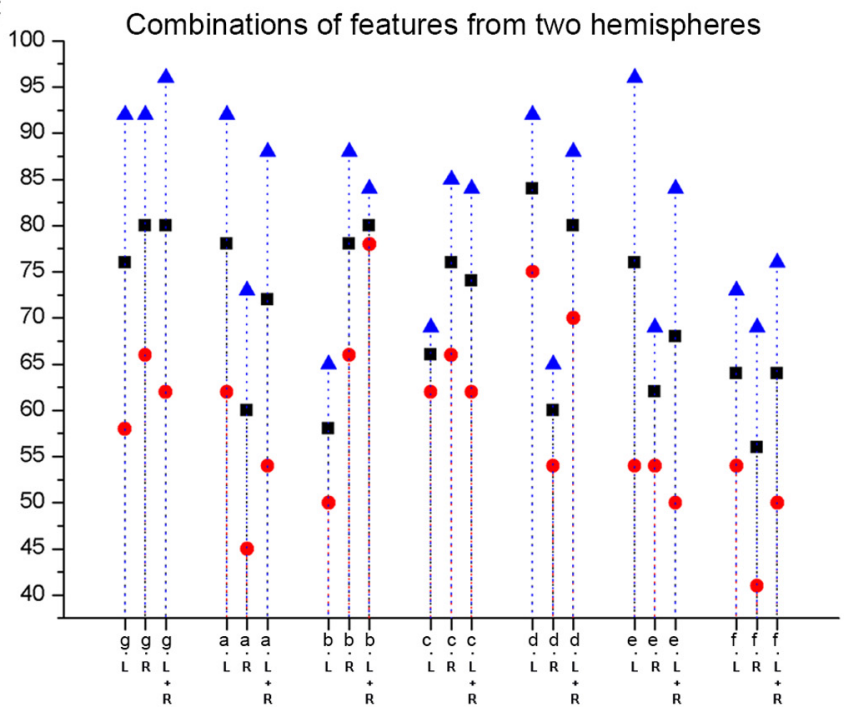

- accuracy (\%)

- sensitivity $(\%)$

- specificity(\%)

a : cortical thickness

b : sulcal depth

c : surface area

$\mathrm{d}:$ metric distortion

e : mean curvature

$f: G M$ volume

\section{$g:$ All six features}

Figure 2. Classifications of the combinations of the different morphological features. The classification results of the combinations of any two morphological features are presented for the left hemisphere $(\boldsymbol{A})$ and the right hemisphere $(\boldsymbol{B})$. The classification results of the combined features from the two hemispheres are shown in $\boldsymbol{C}$.

feature combined with the MMSE and MoCA classification. We did determine that the classification results that used the MMSE and MoCA scores were better compared with the morphologybased features.

\section{Discrimination maps of aMCI-specific abnormalities}

A discrimination map is a spatial representation of the SVM weight vector and represents a spatially distributed pattern that shows the relative contribution of each voxel to the decision function. Due to the multivariate nature of the SVM, each region in the discrimination maps should be considered in the context of the entire discriminating pattern. Here, we used all combined six morphological features to construct the classifiers; the brain regions identified by the weight vector are shown in Figure 6, and a summary description can be found in Tables 6 (left hemisphere) and 7 (right hemisphere).

\section{Cortical thickness}

In the left hemisphere, the high-discriminative patterns for cortical thickness in classifying the two groups are located in the supramarginal gyrus, the middle frontal gyrus, the lingual, para- hippocampal, and isthmus cingulate gyrus. In the right hemisphere, the high-discriminative pattern for cortical thickness was involved in the superior temporal sulcus, the superior frontal gyrus, the postcentral gyrus, the intraparietal and parietal transverse sulcus, and the subcentral region (Fig. 6A).

\section{Sulcal depth}

The left parieto-occipital sulcus, the superior frontal sulcus, the precentral gyrus, and the middle temporal gyrus showed high weights in the discrimination map. The right inferior frontal sulcus, the parieto-occipital sulcus, the postcentral gyrus, the pars opercularis, the angular gyrus, the inferior temporal gyrus, the precentral gyrus, the superior parietal gyrus, the superior temporal sulcus, and the central sulcus were observed in relatively large clusters with generally high weights (Fig. 6B).

\section{Surface area}

The regions that showed high-weight values included the superior temporal gyrus, the lingual gyrus, and the superior frontal gyrus in the left hemisphere. In the right hemisphere, the highweight value regions were the angular gyrus, the lateral orbito- 


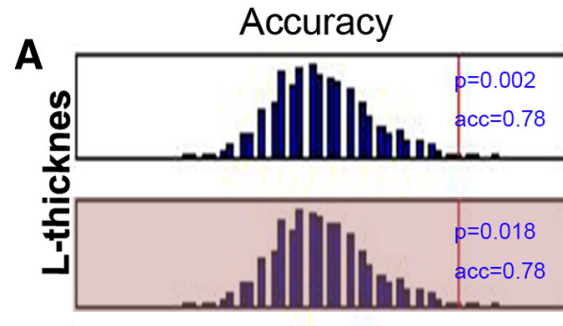

B

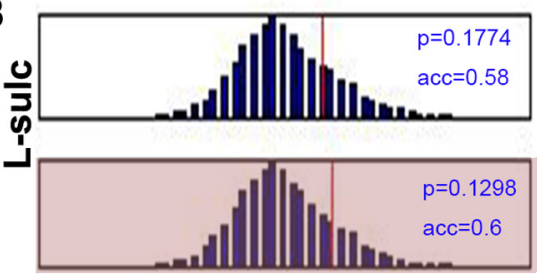

C

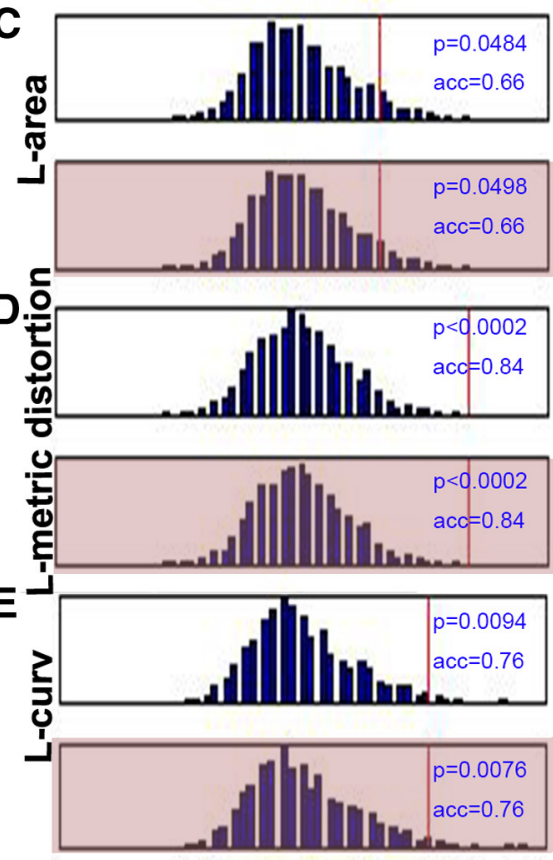

F

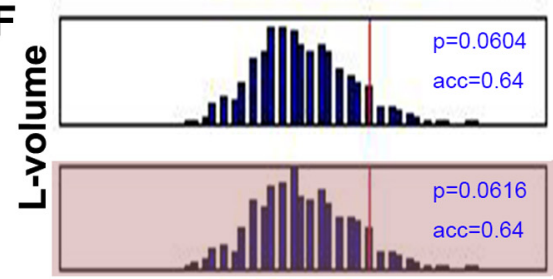

G

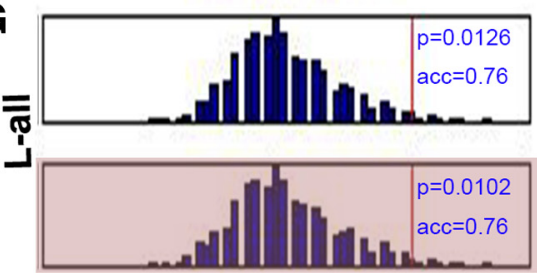

Sensitivity
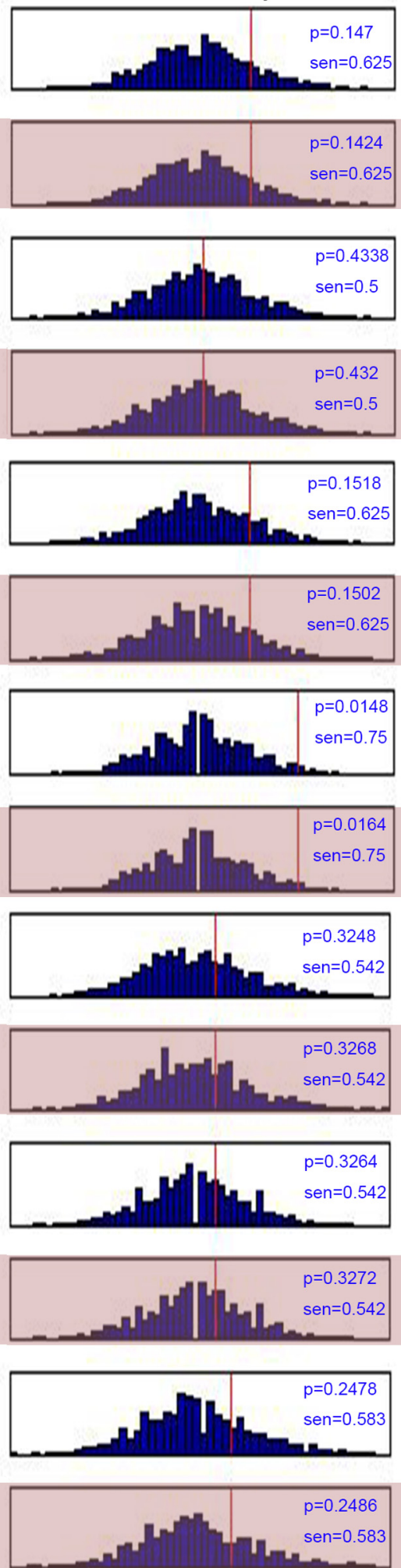
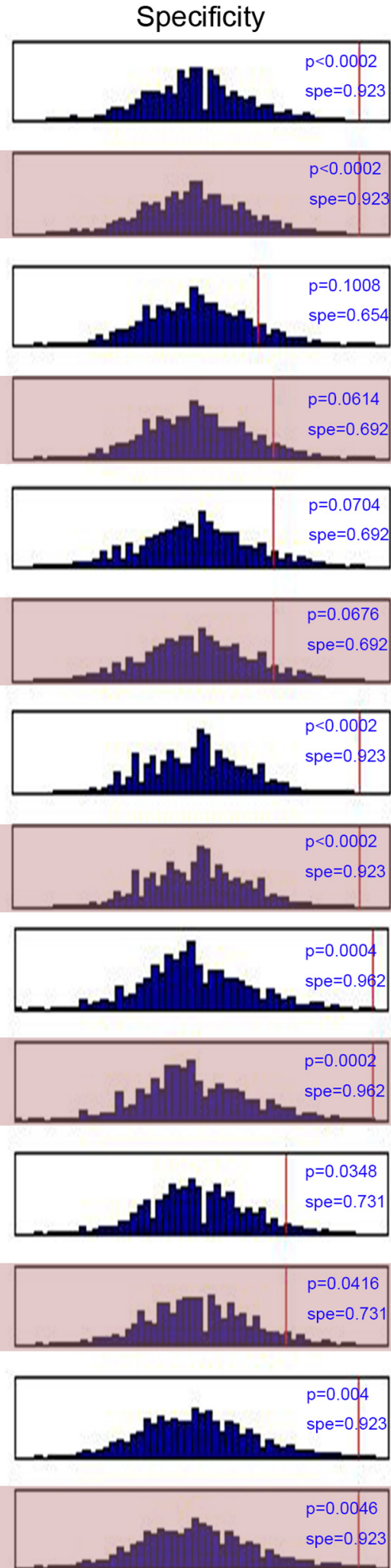

Figure 3. Null histograms generated by 5000 permutation tests of the SVM classification based on the cortical thickness $(\boldsymbol{A})$, sulcal depth $(\boldsymbol{B})$, surface area $(\boldsymbol{C})$, metric distortion $(\boldsymbol{D})$, mean curvature $(\boldsymbol{E})$, GM volume $(\boldsymbol{F})$, and all parameters $(\boldsymbol{G})$ for the left hemisphere. The null histograms from left to right represent the accuracy, sensitivity, and specificity, respectively. The odd-numbered rows represent the classifications that were performed only using morphological features, while the even-numbered (pink background) rows represent the classifications that used a combination of the morphological features and the MMSE and MoCA scores. 

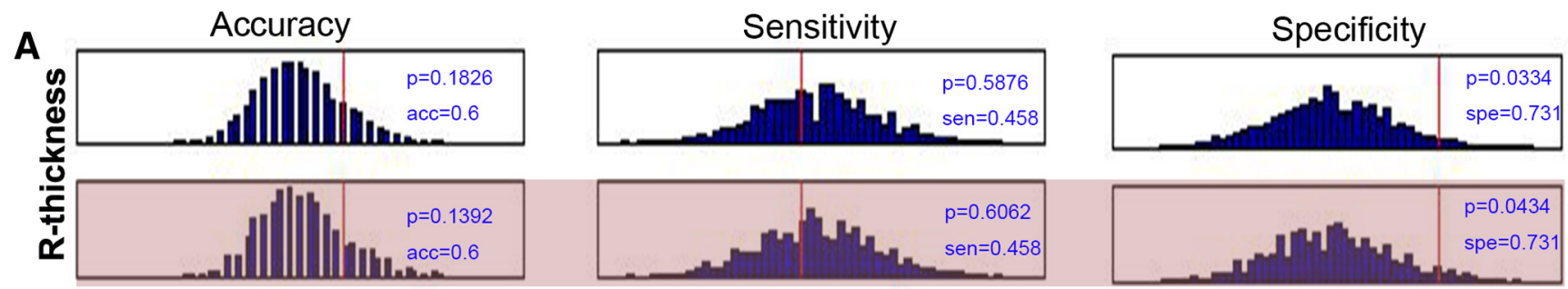

B

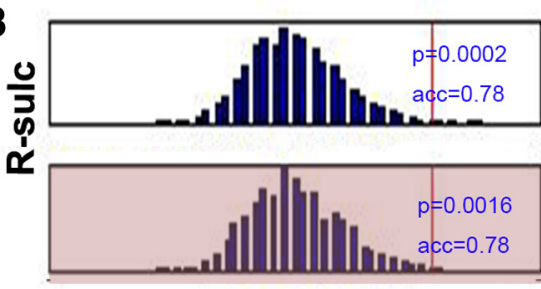

C

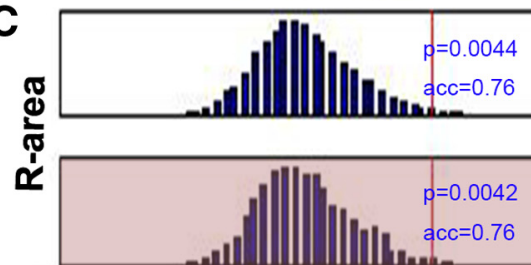

D.ำ

高

E

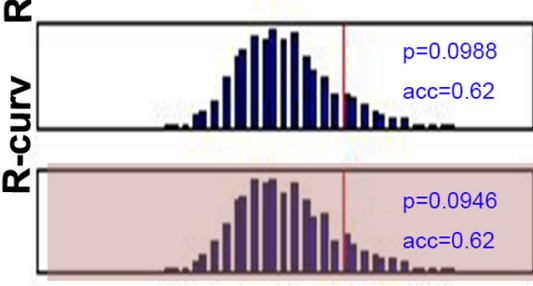

F

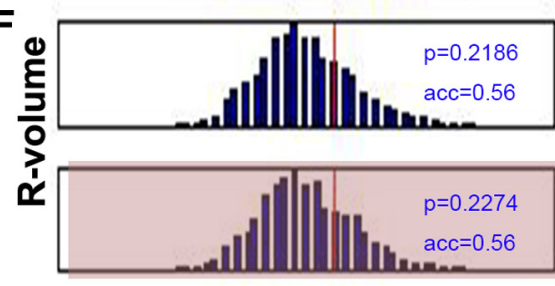

G

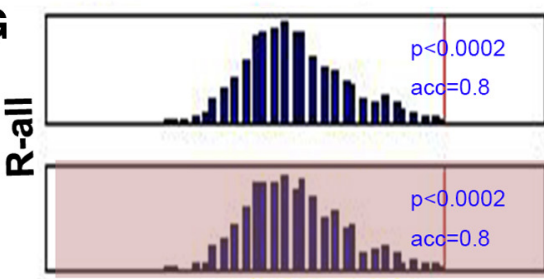

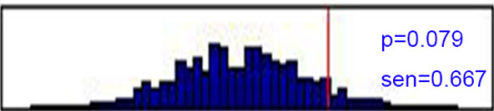
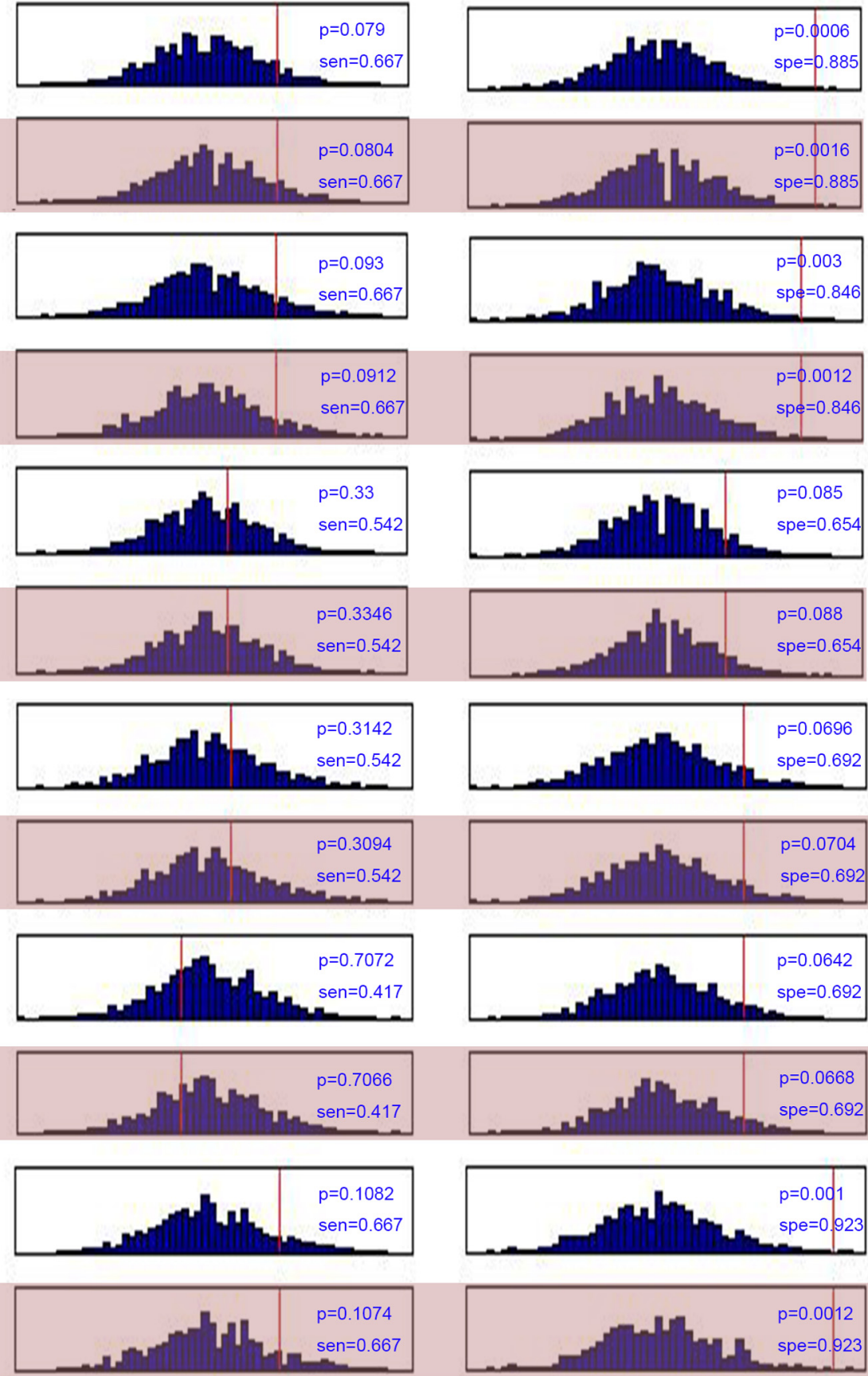

Figure 4. Null histograms generated by 5000 permutation tests of the SVM classification based on the cortical thickness $(\boldsymbol{A})$, sulcal depth $(\boldsymbol{B})$, surface area $(\boldsymbol{C})$, metric distortion $(\boldsymbol{D})$, mean curvature $(\boldsymbol{E})$, GM volume $(\boldsymbol{F})$, and all parameters $(\boldsymbol{G})$ for the right hemisphere. The null histograms from left to right represent the accuracy, sensitivity, and specificity, respectively. The odd-numbered rows represent the classifications that were performed only using the morphological features, while the even-numbered (pink background) rows represent the classifications that used a combination of the morphological features and the MMSE and MoCA scores. 

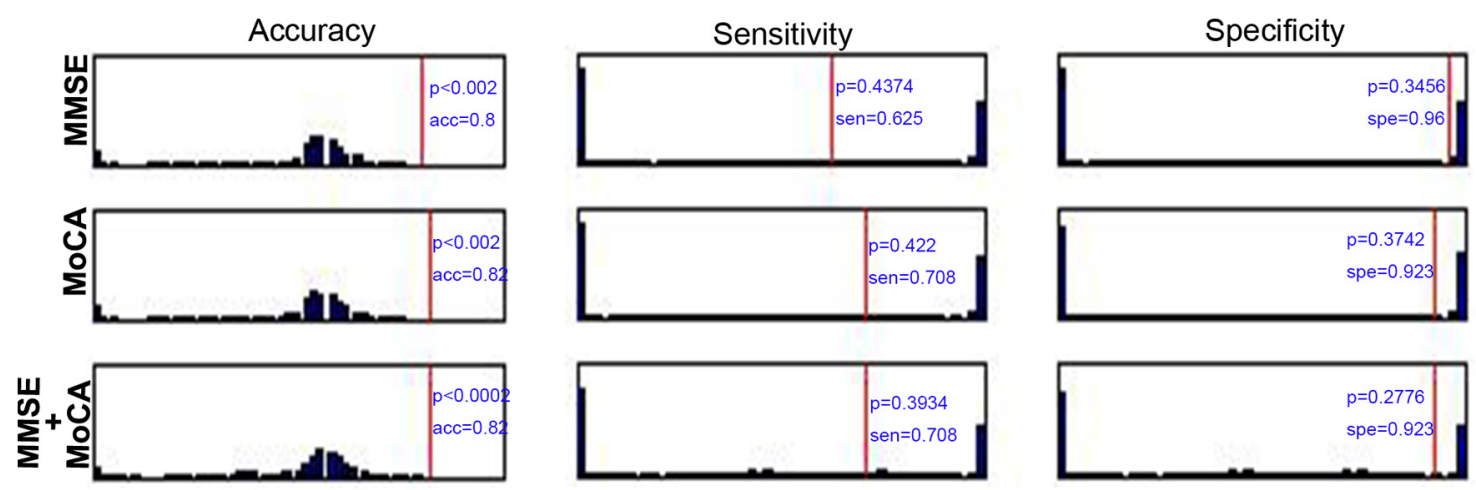

Figure 5. Null histograms generated by 5000 permutation tests of the SVM classification based on the MMSE and MoCA scores. The null histograms from left to right represent the accuracy, sensitivity, and specificity, respectively. The first two rows represent the classification with only the MMSE or MoCA score. The third row represents the classification that used the combination of the two scores.

frontal sulcus, the inferior parietal sulcus, the middle frontal rostral region, the pars triangularis gyrus, the central sulcus, the temporal pole, the superior temporal sulcus, and the precentral gyrus (Fig. 6C).

\section{Metric distortion}

In the left hemisphere, the superior temporal sulcus, the occipital pole, the parietal occipital sulcus, the postcentral gyrus, the lateral orbital sulcus, and the supramarginal gyrus had high-weight values. In the right hemisphere, the regions that showed the highweight values were found in the cuneus gyrus, the cingulate gyrus, the frontal gyrus, the postcentral gyrus, and the precentral gyrus (Fig. 6D).

\section{Mean curvature}

The left superior occipital gyrus, the transverse temporal gyrus, the pars opercularis, and the parahippocampus showed the high weights in the discrimination map of the mean curvature. Right high-weight value regions were observed in the central sulcus, the superior occipital gyrus, the calcarine sulcus, and the superior frontal gyrus (Fig. 6E).

\section{GM volume}

We identified a high weight in the left superior temporal gyrus in the GM volume discrimination map. In the right hemisphere, the high discriminative pattern was found in the temporal pole, the central sulcus, and the middle frontal rostral gyrus (Fig. $6 F$ ).

\section{ROI analysis}

In this study, we selected the parahippocampal gyrus and the entorhinal area as the ROIs because many studies had shown cortical thickness or GM volume decline in the two regions in aMCI subjects (Karas et al., 2004; Pennanen et al., 2004). The morphological profiles for the two ROIs are shown in the box plot of Figure $7 A$ and $B$. The weight for each ROI was derived by averaging the weight vector scores across the vertices within the ROI for each morphometric parameter. We observed that different cortical parameters had different contributions for each region in discriminating the two groups. For the parahippocampal gyrus, the high weights were observed for cortical thickness, which had a mean weight value of -2.71 (negative sign indicates that aMCI had lower parameter values compared with NC), but low weights were observed in the surface area $(-1.00)$, the metric distortion $(-1.35)$, and the mean curvature (1.01; Fig. 7A). A similar profile was also seen in the entorhinal area. Here, the weight values were very low for the cortical thickness $(-0.39)$, the sulcal depth $(-0.79)$, the sur- face area $(-0.88)$, the metric distortion $(-0.34)$, and the GM volume $(-0.81)$, while high-discrimination weights were observed in the mean curvature $(-2.70$; Fig. $7 B)$. The ROI analysis indicated that the discrimination maps were spatially distributed, and the different measures showed different weight values from region to region.

\section{Discussion}

In this study, we used a multiparameter classification approach that combined six cortical features to investigate the subtle and spatially distributed GM abnormalities in aMCI patients. The accuracy of the SVM in classifying the aMCI and NC groups was $76 \%$ in the left hemisphere and $80 \%$ in the right hemisphere. The high-classification weight regions, which were generated in a multivariate way under the framework established by the SVM, were spatially distributed and largely nonoverlapping, predominately located in the temporal, frontal, and parietal regions. The weight value analysis of the chosen ROIs (the parahippocampal gyrus and the entorhinal cortex) also showed that the different morphological features had unique contributions to the classification of the aMCI and NC groups. These results suggested that the neuroanatomical changes of aMCI were truly multidimensional. The weight value maps detected using the SVM may help further exploration of the genetic and neuropathological underpinnings of aMCI.

The high SVM accuracy is the cornerstone of this study. In the left hemisphere, the SVM correctly classified $76 \%$ of cases overall using six cortical features; in the right hemisphere, the accuracy was $80 \%$. When combining any two features for the left hemisphere, the highest classification accuracy $(84 \%)$ was obtained by combining the metric distortion and the cortical thickness features. This accuracy is comparable to previous reports (Westman et al., 2011; Wolz et al., 2011; Zhang et al., 2011; Liu et al., 2012; O’Dwyer et al., 2012; Park et al., 2012; Zhou et al., 2012). An SVM study that used combined features (cortical thickness and sulcus depth) had a 69\% accuracy in classifying the MCI and NC groups, and the accuracy reached $79 \%$ after the principle component analysis for the feature selection (Park et al., 2012). Zhang et al. (2011) used a kernel combination method based on the SVM to classify MCI patients and controls by using multimodality data (MRI, PET, and CSF) and obtained a classification accuracy of $76.4 \%$. These classification accuracies were also consistent with the behaviorally guided diagnostic tools (MMSE and MoCA), which had accuracies of $\sim 80 \%$ in this study. Our classification results indicated that the cortical morphological features could 

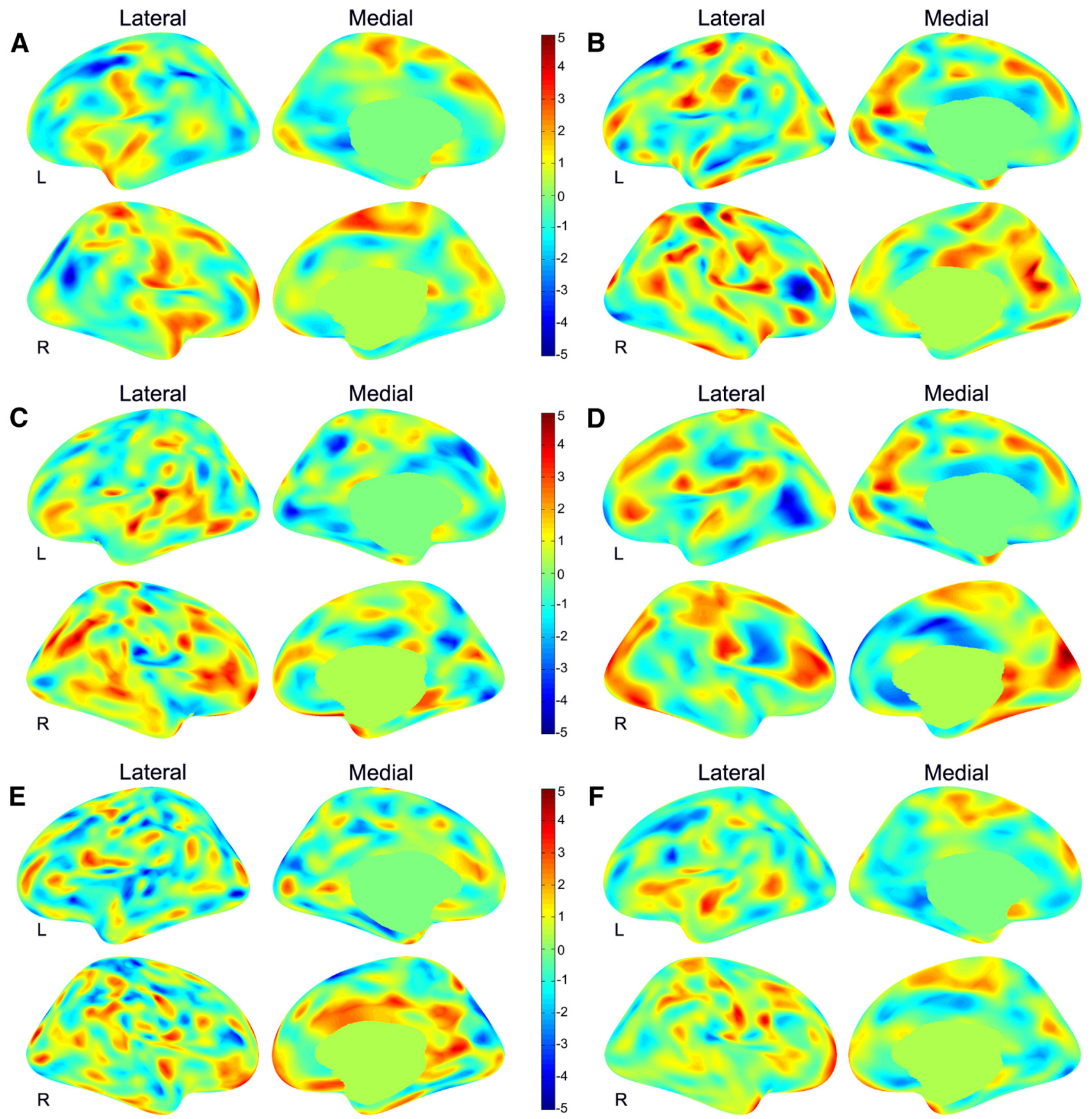

Figure 6. Discrimination maps for the six different morphometric features in the left $(\mathrm{L})$ and right $(\mathrm{R})$ hemispheres. The color maps represent the weight vector on the basis of the six modality classification for the cortical thickness $(\boldsymbol{A})$, sulcal depth $(\boldsymbol{B})$, surface area $(\boldsymbol{C})$, metric distortion $(\boldsymbol{D})$, mean curvature $(\boldsymbol{E})$, and GM volume $(\boldsymbol{F})$. The positive weights are displayed in red and the negative weights are displayed in blue.

potentially be used to construct an effective classifier in discriminating aMCI patients from normal controls.

The SVM is a multivariate method that considers interregional correlations, and it can be used to identify complex neuroanatomical changes. In the present study, each region in the discrimination maps should be considered in the context of the entire pattern of discrimination. The spatial distribution of the weight vector provided information about the contribution of each region to the classification and made it possible to obtain the distributed pattern of a relative deficit or excess in aMCI with respect to the controls. We found cortical thickness reductions in the medial temporal lobe regions, such as the parahippocampus and the lingual and isthmus cingulate gyrus. These findings were in line with previous studies (Karas et al., 2004; Pennanen et al., 2004; Singh et al., 2006; Wang et al., 2009). We also observed that the depth decreased in the left middle temporal sulcus in the aMCI patients compared with the controls. Our result was similar to a study by Im et al. (2008), and they reported aMCI showed shallower sulcal depth in the temporal lobe. In addition, we found that aMCI had a significant GM volume reduction in the left lateral superior temporal gyrus. Previous studies have reported a GM volume decline in the hippocampus, the middle temporal gyrus, and the inferior parietal lobe in aMCI subjects (Pen- 
Table 6. Regions that displayed high-discrimination weights between the aMCI and control groups for the measures of the left hemisphere

\begin{tabular}{|c|c|c|c|c|c|c|}
\hline Annotation & $\mathrm{NV}_{\mathrm{txs}}$ & Size $\left(\mathrm{mm}^{2}\right)$ & TalX & TalY & TalZ & Max \\
\hline \multicolumn{7}{|l|}{ Cortical thickness } \\
\hline Supramarginal & 1601 & 645.6 & -45.8 & -47.7 & 41 & -5.00 \\
\hline Caudal middle frontal & 2138 & 1299.5 & -39.2 & 5.3 & 51.4 & -4.05 \\
\hline $\begin{array}{l}\text { Lingual, parahippocampal, } \\
\text { isthmus cingulate } \\
\text { gyrus }\end{array}$ & 800 & 336 & -15.6 & -41.8 & -7.3 & -3.16 \\
\hline \multicolumn{7}{|l|}{ Sulcal depth } \\
\hline Cuneus & 1315 & 700.0 & -16.1 & -68.8 & 15.1 & 4.17 \\
\hline Sulcus superior frontal & 1511 & 960.7 & -22.2 & 17.5 & 53 & -4.08 \\
\hline Gyrus precentral & 1362 & 634.4 & -34.9 & -12 & 60.1 & 4.05 \\
\hline Gyrus middle temporal & 987 & 544.4 & -58.5 & -15.7 & -18.7 & -4.00 \\
\hline \multicolumn{7}{|l|}{ Surface area } \\
\hline Gyrus superior temporal & 1406 & 559.3 & -44.5 & -34.7 & 5.8 & 5.00 \\
\hline Pericalcarine & 883 & 592 & -9.7 & -83.8 & 1.1 & -4.17 \\
\hline Gyrus superior frontal & 1016 & 578.3 & -7.7 & 28.3 & 41.5 & -3.66 \\
\hline \multicolumn{7}{|l|}{ Metric distortion } \\
\hline $\begin{array}{l}\text { Inferior parietal, middle } \\
\text { temporal, lateral } \\
\text { occipital }\end{array}$ & 2504 & 1394.4 & -48.2 & -63.4 & 10.5 & -4.55 \\
\hline Pericalcarine & 1491 & 1207.7 & -13.1 & -97.6 & 1.6 & 4.09 \\
\hline Precuneus & 1662 & 940.1 & -17.1 & -60.4 & 22 & -4.04 \\
\hline Postcentral & 923 & 395.5 & -12.5 & -36.3 & 68.4 & 3.95 \\
\hline Pars triangularis & 970 & 620.1 & -44.3 & 37 & -5.4 & 3.46 \\
\hline Supramarginal & 2166 & 969.3 & -48.6 & -28.2 & 19.3 & 3.25 \\
\hline \multicolumn{7}{|l|}{ Mean curvature } \\
\hline Superior parietal & 1058 & 810.2 & -11.2 & -91.5 & 26.5 & -4.09 \\
\hline Transverse temporal & 1031 & 425.8 & -52.2 & -13.5 & 1.6 & -3.47 \\
\hline \multirow{2}{*}{$\begin{array}{l}\text { Pars opercularis } \\
\text { entorhinal }\end{array}$} & 932 & 380.6 & -39.8 & 6.2 & 21.2 & 3.40 \\
\hline & 986 & 399.5 & -23.5 & -18.7 & -25.1 & -3.37 \\
\hline \multicolumn{7}{|l|}{ GM volume } \\
\hline Superior temporal & 1214 & 601.7 & -57.4 & -1.5 & -4.8 & 3.57 \\
\hline
\end{tabular}

nanen et al., 2004; Bell-McGinty et al., 2005; Fan et al., 2008). The consistencies between our findings and previous studies indicated that the regions that displayed high-classification weights could reflect the differences in the cortical cytoarchitecture and folding patterns between aMCI patients and normal controls and might help the clinical diagnosis of aMCI.

In this study, we found that the classification accuracies varied across different morphometric parameters during the discrimination of the two groups. The high accuracies were obtained when using the metric distortion and cortical thickness in the left hemisphere and the sulcal depth in the right hemisphere in classifying the aMCI and NC groups. Many studies have explored the changes in the cortical thickness (Bell-McGinty et al., 2005; Singh et al., 2006; Seo et al., 2007) and the sulcal depth (Im et al., 2008; Liu et al., 2013) in aMCI subjects. However, metric distortion was seldom used to measure the cerebral changes in aMCI patients. Our study used metric distortion to characterize the cortical surface feature and obtained $84 \%$ classification accuracy in the left hemisphere, which was better compared with the other cortical measures. This finding may indicate that the aMCI patients had a significant overall cortical transformation compared with the normal controls. The existing studies had predominately focused on the GM atrophy in aMCI. Our finding suggested that metric distortion might contain important information to distinguish aMCI patients and normal controls and should receive greater attention in future aMCI studies.

Notably, the different measures reflect different neurophysiological processes, and the region- and parameter-dependent
Table 7. Regions that displayed high-discrimination weights between the aMCI and control groups for the measures of the right hemisphere

\begin{tabular}{|c|c|c|c|c|c|c|}
\hline Annotation & $\mathrm{NV}_{\mathrm{txs}}$ & Size $\left(\mathrm{mm}^{2}\right)$ & TalX & TalY & TalZ & Max \\
\hline \multicolumn{7}{|l|}{ Cortical thickness } \\
\hline Inferior parietal & 1156 & 632.4 & 40 & -61.4 & 21.5 & -4.56 \\
\hline Superior frontal & 2061 & 995.8 & 6.7 & 2 & 65.6 & 3.70 \\
\hline Postcentral & 1424 & 595.0 & 30.3 & -32.4 & 68.5 & 3.65 \\
\hline Superior parietal & 1203 & 618.9 & 22.6 & -62.8 & 35 & -3.56 \\
\hline Postcentral & 2576 & 1082.4 & 49.3 & -12.6 & 18.1 & 3.42 \\
\hline \multicolumn{7}{|l|}{ Sulcal depth } \\
\hline Pars triangularis & 1743 & 959 & 42.3 & 31.6 & 11.2 & -5.00 \\
\hline Precuneus & 1519 & 857.8 & 12.2 & -62.8 & 26.2 & 4.95 \\
\hline Postcentral & 1534 & 647.8 & 40 & -32.7 & 63.3 & 4.66 \\
\hline Pars opercularis & 1500 & 756.9 & 52.4 & 7.9 & 3.8 & 4.47 \\
\hline Inferior parietal & 1212 & 634.6 & 53.8 & -52.7 & 33.9 & 4.08 \\
\hline Inferior temporal & 961 & 597.0 & 56.1 & -28.9 & -28 & 3.86 \\
\hline Precentral & 1161 & 586.9 & 54.9 & -3.2 & 34.5 & 3.55 \\
\hline Superior parietal & 1455 & 656.9 & 21.2 & -58.5 & 64.1 & 3.52 \\
\hline Inferior parietal & 869 & 384.3 & 46.1 & -57 & 14.7 & 3.36 \\
\hline Precentral & 1090 & 427.2 & 20.1 & -29.6 & 53.4 & -3.23 \\
\hline \multicolumn{7}{|l|}{ Surface area } \\
\hline Inferior parietal & 2669 & 1335.9 & 47.9 & -60.9 & 31.4 & 4.64 \\
\hline Lateral orbitofrontal & 1422 & 639.7 & 14.6 & 20.5 & -13.9 & 4.34 \\
\hline Inferior parietal & 912 & 449.2 & 32.2 & -70.2 & 20.2 & 4.16 \\
\hline $\begin{array}{l}\text { Rostral middle } \\
\text { frontal }\end{array}$ & 868 & 665.4 & 29.3 & 56.6 & -8.4 & 4.10 \\
\hline Pars triangularis & 2678 & 1438.6 & 46.8 & 24.9 & 7 & 3.94 \\
\hline Precentral & 1037 & 421.6 & 15.3 & -28.2 & 57.6 & 3.76 \\
\hline Temporal pole & 1006 & 545.3 & 30.5 & 10.5 & -34.4 & 3.62 \\
\hline Middle temporal & 1787 & 738.2 & 47.9 & -32.7 & -7.9 & 3.56 \\
\hline Precentral & 933 & 380.2 & 55.8 & 4.9 & 13.7 & -3.17 \\
\hline \multicolumn{7}{|l|}{ Metric distortion } \\
\hline Cuneus & 10382 & 6808.3 & 7.3 & -83.3 & 24.5 & 5.00 \\
\hline Posterior cingulate & 2125 & 827.8 & 10.6 & -4.2 & 42.8 & -4.80 \\
\hline Superior frontal & 1523 & 1002.1 & 15.6 & 54.5 & 24.2 & -4.11 \\
\hline $\begin{array}{l}\text { Rostral middle } \\
\text { frontal }\end{array}$ & 3047 & 1863.8 & 38.3 & 48.7 & 15.9 & 3.97 \\
\hline Postcentral & 1890 & 783.5 & 60.2 & -18.5 & 19 & 3.63 \\
\hline Isthmus cingulate & 1655 & 630.5 & 10.6 & -48.5 & 5.2 & 3.38 \\
\hline Precentral & 2118 & 1056.8 & 44.6 & 3.7 & 21.6 & -3.18 \\
\hline \multicolumn{7}{|l|}{ Mean curvature } \\
\hline Precentral & 1763 & 771.8 & 21.3 & -23.2 & 59.6 & -4.12 \\
\hline Superior parietal & 974 & 613.2 & 14.8 & -87.2 & 33.8 & -4.10 \\
\hline Precuneus & 1480 & 654.0 & 26.5 & -65.3 & 6.9 & 4.10 \\
\hline Superior frontal & 2621 & 1925.1 & 13.2 & 57.9 & 21.1 & 3.87 \\
\hline \multicolumn{7}{|l|}{ GM volume } \\
\hline Temporal pole & 1002 & 606.6 & 37.3 & 16.2 & -34.4 & 4.18 \\
\hline Precentral & 895 & 404.9 & 48.7 & -7.6 & 24.5 & 3.97 \\
\hline $\begin{array}{l}\text { Rostral middle } \\
\text { frontal }\end{array}$ & 2521 & 1797.1 & 23.6 & 58.9 & 6.4 & 3.78 \\
\hline
\end{tabular}

The classifiers were constructed by combining all six morphological features.

variations may reflect the pathology/ pathophysiology of aMCI. For example, the cortical thickness may reflect the dendritic arborization (Huttenlocher, 1990) or the changing myelination at the gray/white matter interface (Sowell et al., 2004). In contrast, the surface area is influenced by the division of progenitor cells in the embryological periventricular area and is associated with the number of minicolumns (Rakic, 1988). The cortical thickness and surface area reflect different neurobiological processes and are associated with different genetic mechanisms (Panizzon et al., 2009). In contrast, the geometric differences, such as the average convexity, the metric distortion, and the sulcal depth, measure different aspects of cortical geometry and are predominantly linked with the development of neuronal connections and the cortical pattern of connectivity (Van Essen, 1997). Our findings 


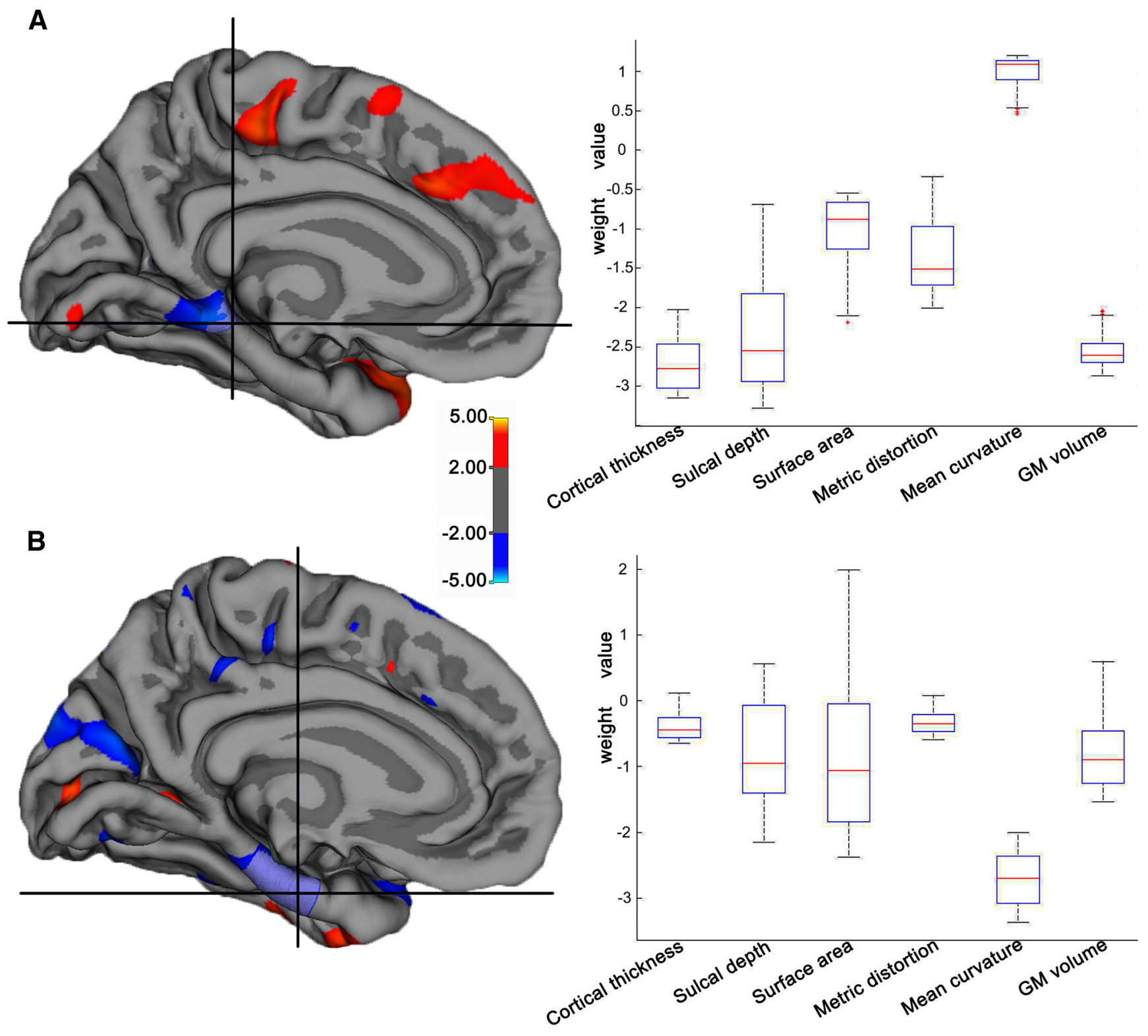

Figure 7. The ROl analysis of the weight values. $\boldsymbol{A}$, The cluster of the cortical thickness in the parahippocampal gyrus, left hemisphere. $\boldsymbol{B}$, The cluster of the mean curvature in the entorhinal, left hemisphere. The box figure shows the average weight values in the ROI for the different morphometric parameters. The weights were identified by the SVM model based on the combined surface features, and thus show the relative contributions of the parameters in this ROl.

in the discrimination map might indicate that the spatial distribution of the weight vector across the whole brain reflects multiple genetic and/or neurobiological processes and requires further investigation.

Further ROI analysis showed that the different cortical parameters played different roles for each region in discriminating the two groups. We found that the mean curvature had the largest weight in discriminating the aMCI and $\mathrm{NC}$ groups in the entorhinal cortex. A previous study suggested that some geometric measures, such as metric distortion and mean curvature, may reflect the cortical folding pattern and vary with changes in the intrinsic and extrinsic connectivity (Van Essen, 1997). Some studies on early AD have reported consistent findings that the neurofibrillary tangle distribution (Delacourte et al., 1999; Price et al., 1999; Lee et al., 2009; Cherubini et al., 2010) and the fractal anisotropy of the white matter (Bai et al., 2009) were initially restricted to the entorhinal cortices, but subsequently spread to the higher order temporoparietal association cortices (Thompson et al., 2003; van der Flier et al., 2011). Thus, we speculated that the abnormal changes of curvature in the entorhinal cortex in the aMCI patients could be related to the pathological changes in the white matter, and these changes might be much larger compared with the other cortical parameters. In addition, we also found that the cortical thickness in the parahippocampal gyrus showed the largest weight value compared with the remaining parameters in discriminating the two groups. Our results were consistent with previous studies. For example, Devanand et al. (2007) reported a volume reduction of the parahippocampal gyrus in MCI patients. Lerch et al. (2005b) reported a significant cortical thickness decline in the parahippocampal gyrus in AD. Because cortical thickness correlates with the number of neurons within an ontogenetic column, our results may indicate the main cellular changes in the parahippocampal gyrus in aMCI. 
Some issues need to be considered. First, the cerebral pathological changes in aMCI would be affected by the subtype, the APOE genotype, and the developmental stage. To clarify the multidimensional changes in aMCI patients, it is better to control for the aMCI subjects by studying patients with the same aMCI subtype, APOE genotype, and stage. Second, although the SVM could solve high-dimension, small sample classification problems, the small sample size still had a negative effect on classifying the results (Hua et al., 2005; Kuo et al., 2007; Ramírez et al., 2010). A future study based on a large sample size will help reveal the more complex multidimensional cortical differences in aMCI. Third, only structural MRI was involved in this study; the combination of functional and quantitative MR techniques as well as $\beta$-amyloid (A $\beta$ ) 1-42 and total tau in the CSF is expected to be more capable in differentiating aMCI and $\mathrm{NC}$ with the additional information provided for the microstructure integrity, energy metabolism, and tissue characterization.

In conclusion, this study used an advanced multivariate method to classify aMCI and NC and determined the spatially complex multidimensional patterns of GM differences in aMCI. The discrimination maps generated by our study will help further the study of aMCI etiologies.

\section{References}

Apostolova LG, Steiner CA, Akopyan GG, Dutton RA, Hayashi KM, Toga AW, Cummings JL, Thompson PM (2007) Three-dimensional gray matter atrophy mapping in mild cognitive impairment and mild Alzheimer disease. Arch Neurol 64:1489-1495. Medline

Bai F, Zhang Z, Watson DR, Yu H, Shi Y, Yuan Y, Qian Y, Jia J (2009) Abnormal integrity of association fiber tracts in amnestic mild cognitive impairment. J Neurol Sci 278:102-106. CrossRef Medline

Bell-McGinty S, Lopez OL, Meltzer CC, Scanlon JM, Whyte EM, Dekosky ST, Becker JT (2005) Differential cortical atrophy in subgroups of mild cognitive impairment. Arch Neurol 62:1393-1397. CrossRef Medline

Bendfeldt K, Klöppel S, Nichols TE, Smieskova R, Kuster P, Traud S, MuellerLenke N, Naegelin Y, Kappos L, Radue EW, Borgwardt SJ (2012) Multivariate pattern classification of gray matter pathology in multiple sclerosis. Neuroimage 60:400-408. CrossRef Medline

Burges CJC (1998) A tutorial on support vector machines for pattern recognition. Data Min Knowl Disc 2:121-167. CrossRef

Cherubini A, Péran P, Spoletini I, Di Paola, Di Iulio F, Hagberg GE, Sancesario G, Gianni W, Bossù P, Caltagirone C, Sabatini U, Spalletta G (2010) Combined volumetry and DTI in subcortical structures of mild cognitive impairment and Alzheimer's disease patients. J Alzheimers Dis 19:12731282. CrossRef Medline

Dale AM, Fischl B, Sereno MI (1999) Cortical surface-based analysis. I. Segmentation and surface reconstruction. Neuroimage 9:179-194. CrossRef Medline

Davatzikos C, Fan Y, Wu X, Shen D, Resnick SM (2008) Detection of prodromal Alzheimer's disease via pattern classification of magnetic resonance imaging. Neurobiol Aging 29:514-523. CrossRef Medline

Delacourte A, David JP, Sergeant N, Buée L, Wattez A, Vermersch P, Ghozali F, Fallet-Bianco C, Pasquier F, Lebert F, Petit H, Di Menza C (1999) The biochemical pathway of neurofibrillary degeneration in aging and Alzheimer's disease. Neurology 52:1158-1165. CrossRef Medline

Devanand DP, Pradhaban G, Liu X, Khandji A, De Santi S, Segal S, Rusinek H, Pelton GH, Honig LS, Mayeux R, Stern Y, Tabert MH, de Leon MJ (2007) Hippocampal and entorhinal atrophy in mild cognitive impairment: prediction of Alzheimer disease. Neurology 68:828-836. CrossRef Medline

Ecker C, Marquand A, Mourão-Miranda J, Johnston P, Daly EM, Brammer MJ, Maltezos S, Murphy CM, Robertson D, Williams SC, Murphy DG (2010) Describing the brain in autism in five dimensions-magnetic resonance imaging-assisted diagnosis of autism spectrum disorder using a multiparameter classification approach. J Neurosci 30:10612-10623. CrossRef Medline

Fan Y, Batmanghelich N, Clark CM, Davatzikos C (2008) Spatial patterns of brain atrophy in MCI patients identified via high-dimensional pattern classification predict subsequent cognitive decline. Neuroimage 39:17311743. CrossRef Medline
Fischl B, Dale AM (2000) Measuring the thickness of the human cerebral cortex from magnetic resonance images. Proc Natl Acad Sci U S A 97: 11050-11055. CrossRef Medline

Fischl B, Sereno MI, Dale AM (1999) Cortical surface-based analysis. II: Inflation, flattening, and a surface-based coordinate system. Neuroimage 9:195-207. CrossRef Medline

Fischl B, Salat DH, Busa E, Albert M, Dieterich M, Haselgrove C, van der Kouwe A, Killiany R, Kennedy D, Klaveness S, Montillo A, Makris N, Rosen B, Dale AM (2002) Whole brain segmentation: automated labeling of neuroanatomical structures in the human brain. Neuron 33:341355. CrossRef Medline

Fischl B, Salat DH, van der Kouwe AJ, Makris N, Ségonne F, Quinn BT, Dale AM (2004a) Sequence-independent segmentation of magnetic resonance images. Neuroimage 23:S69-S84. CrossRef Medline

Fischl B, van der Kouwe A, Destrieux C, Halgren E, Ségonne F, Salat DH, Busa E, Seidman LJ, Goldstein J, Kennedy D, Caviness V, Makris N, Rosen B, Dale AM (2004b) Automatically parcellating the human cerebral cortex. Cereb Cortex 14:11-22. CrossRef Medline

Frisoni GB, Pievani M, Testa C, Sabattoli F, Bresciani L, Bonetti M, Beltramello A, Hayashi KM, Toga AW, Thompson PM (2007) The topography of grey matter involvement in early and late onset Alzheimer's disease. Brain 130:720-730. CrossRef Medline

Hua J, Xiong Z, Lowey J, Suh E, Dougherty ER (2005) Optimal number of features as a function of sample size for various classification rules. Bioinformatics 21:1509-1515. CrossRef Medline

Huttenlocher PR (1990) Morphometric study of human cerebral cortex development. Neuropsychologia 28:517-527. CrossRef Medline

Im K, Lee JM, Seo SW, Hyung Kim S, Kim SI, Na DL (2008) Sulcal morphology changes and their relationship with cortical thickness and gyral white matter volume in mild cognitive impairment and Alzheimer's disease. Neuroimage 43:103-113. CrossRef Medline

Johnson DE (2005) Applied multivariate methods for data analysts. Beijing: Higher Education.

Jovicich J, Czanner S, Greve D, Haley E, van der Kouwe A, Gollub R, Kennedy D, Schmitt F, Brown G, Macfall J, Fischl B, Dale A (2006) Reliability in multi-site structural MRI studies: effects of gradient nonlinearity correction on phantom and human data. Neuroimage 30:436-443. CrossRef Medline

Karas GB, Scheltens P, Rombouts SA, Visser PJ, van Schijndel RA, Fox NC, Barkhof F (2004) Global and local gray matter loss in mild cognitive impairment and Alzheimer's disease. Neuroimage 23:708-716. CrossRef Medline

Klöppel S, Stonnington CM, Chu C, Draganski B, Scahill RI, Rohrer JD, Fox NC, Jack CR Jr, Ashburner J, Frackowiak RS (2008) Automatic classification of MR scans in Alzheimer's disease. Brain 131:681-689. CrossRef Medline

Kuo B-C, Chang KY (2007) Feature extractions for small sample size classification problem. IEEE T Geosci Remote Sensing 45:756-764. CrossRef

Lee DY, Fletcher E, Martinez O, Ortega M, Zozulya N, Kim J, Tran J, Buonocore M, Carmichael O, DeCarli C (2009) Regional pattern of white matter microstructural changes in normal aging, $\mathrm{MCI}$, and $\mathrm{AD}$. Neurology 73:1722-1728. CrossRef Medline

Lerch JP, Evans AC (2005a) Cortical thickness analysis examined through power analysis and a population simulation. Neuroimage 24:163-173. CrossRef Medline

Lerch JP, Pruessner JC, Zijdenbos A, Hampel H, Teipel SJ, Evans AC (2005b) Focal decline of cortical thickness in Alzheimer's disease identified by computational neuroanatomy. Cereb Cortex 15:995-1001. Medline

Liu M, Zhang D, Shen D (2012) Ensemble sparse classification of Alzheimer's disease. Neuroimage 60:1106-1116. CrossRef Medline

Liu T, Sachdev PS, Lipnicki DM, Jiang J, Cui Y, Kochan NA, Reppermund S, Trollor JN, Brodaty H, Wen W (2013) Longitudinal changes in sulcal morphology associated with late-life aging and MCI. Neuroimage 74: 337-342. CrossRef Medline

Long X, Zhang L, Liao W, Jiang C, Qiu B (2013) Distinct laterality alterations distinguish mild cognitive impairment and alzheimer's disease from healthy aging: statistical parametric mapping with high resolution MRI. Hum Brain Mapp 34:3400-3410. CrossRef Medline

Mourão-Miranda J, Reynaud E, McGlone F, Calvert G, Brammer M (2006) The impact of temporal compression and space selection on SVM analysis of single-subject and multi-subject fMRI data. Neuroimage 33:10551065. CrossRef Medline 
O’Dwyer L, Lamberton F, Bokde AL, Ewers M, Faluyi YO, Tanner C, Mazoyer B, O'Neill D, Bartley M, Collins DR, Coughlan T, Prvulovic D, Hampel H (2012) Using support vector machines with multiple indices of diffusion for automated classification of mild cognitive impairment. PLoS One 7:e32441. CrossRef Medline

Panizzon MS, Fennema-Notestine C, Eyler LT, Jernigan TL, Prom-Wormley E, Neale M, Jacobson K, Lyons MJ, Grant MD, Franz CE, Xian H, Tsuang M, Fischl B, Seidman L, Dale A, Kremen WS (2009) Distinct genetic influences on cortical surface area and cortical thickness. Cereb Cortex 19:2728-2735. CrossRef Medline

Park H, Yang JJ, Seo J, Lee JM (2012) Dimensionality reduced cortical features and their use in the classification of Alzheimer's disease and mild cognitive impairment. Neurosci Lett 529:123-127. CrossRef Medline

Pennanen C, Kivipelto M, Tuomainen S, Hartikainen P, Hänninen T, Laakso MP, Hallikainen M, Vanhanen M, Nissinen A, Helkala EL, Vainio P, Vanninen R, Partanen K, Soininen H (2004) Hippocampus and entorhinal cortex in mild cognitive impairment and early AD. Neurobiol Aging 25:303-310. CrossRef Medline

Petersen RC (2003) Mild cognitive impairment: aging to Alzheimer's disease. New York: Oxford UP.

Petersen RC, Smith GE, Waring SC, Ivnik RJ, Tangalos EG, Kokmen E (1999) Mild cognitive impairment: clinical characterization and outcome. Arch Neurol 56:303-308. CrossRef Medline

Petersen RC, Doody R, Kurz A, Mohs RC, Morris JC, Rabins PV, Ritchie K, Rossor M, Thal L, Winblad B (2001a) Current concepts in mild cognitive impairment. Arch Neurol 58:1985-1992. CrossRef Medline

Petersen RC, Stevens JC, Ganguli M, Tangalos EG, Cummings JL, DeKosky ST (2001b) Practice parameter: early detection of dementia: mild cognitive impairment (an evidence-based review). Report of the Quality Standards Subcommittee of the American Academy of Neurology. Neurology 56:1133-1142. CrossRef Medline

Price JL, Morris JC (1999) Tangles and plaques in nondemented aging and "preclinical" Alzheimer's disease. Ann Neurol 45:358-368. CrossRef Medline

Rakic P (1988) Defects of neuronal migration and the pathogenesis of cortical malformations. Prog Brain Res 73:15-37. CrossRef Medline

Ramírez J, Górriz JM, Segovia F, Chaves R, Salas-Gonzalez D, López M, Alvarez I, Padilla P (2010) Computer aided diagnosis system for the Alzheimer's disease based on partial least squares and random forest SPECT image classification. Neurosci Lett 472:99-103. CrossRef Medline

Schoelkopf M, Smola A (2002) Learning with kernels. Cambridge. MA: MIT.

Ségonne F, Dale AM, Busa E, Glessner M, Salat D, Hahn HK, Fischl B (2004) A hybrid approach to the skull stripping problem in MRI. Neuroimage 22:1060-1075. CrossRef Medline

Seo SW, Im K, Lee JM, Kim YH, Kim ST, Kim SY, Yang DW, Kim SI, Cho YS, $\mathrm{Na}$ DL (2007) Cortical thickness in single- versus multiple-domain amnestic mild cognitive impairment. Neuroimage 36:289-297. CrossRef Medline

Singh V, Chertkow H, Lerch JP, Evans AC, Dorr AE, Kabani NJ (2006) Spa- tial patterns of cortical thinning in mild cognitive impairment and Alzheimer's disease. Brain 129:2885-2893. CrossRef Medline

Sowell ER, Thompson PM, Leonard CM, Welcome SE, Kan E, Toga AW (2004) Longitudinal mapping of cortical thickness and brain growth in normal children. J Neurosci 24:8223-8231. CrossRef Medline

Talairach J, Tournoux P (1988) Co-planar stereotaxic atlas of the human brain. New York: Thieme.

Thompson PM, Hayashi KM, de Zubicaray G, Janke AL, Rose SE, Semple J, Herman D, Hong MS, Dittmer SS, Doddrell DM, Toga AW (2003) Dynamics of gray matter loss in Alzheimer's disease. J Neurosci 23:9941005. Medline

van der Flier WM, Pijnenburg YA, Fox NC, Scheltens P (2011) Early-onset versus later-onset Alzheimer's disease: the case of the missing APOE 4 allele. Lancet Neurol 10:280-288. CrossRef Medline

Van Essen DC (1997) A tension-based theory of morphogenesis and compact wiring in the central nervous system. Nature 385:313-318. CrossRef Medline

Vapnik VN (1999) The nature of statistical learning theory. New York: Springer.

Wang H, Golob E, Bert A, Nie K, Chu Y, Dick MB, Mandelkern M, Su MY (2009) Alterations in regional brain volume and individual MRI-guided perfusion in normal control, stable mild cognitive impairment, and MCI-AD converter. J Geriatr Psychiatry Neurol 22:35-45. CrossRef Medline

Westman E, Simmons A, Zhang Y, Muehlboeck JS, Tunnard C, Liu Y, Collins L, Evans A, Mecocci P, Vellas B, Tsolaki M, Kłoszewska I, Soininen H, Lovestone S, Spenger C, Wahlund LO (2011) Multivariate analysis of MRI data for Alzheimer's disease, mild cognitive impairment and healthy controls. Neuroimage 54:1178-1187. CrossRef Medline

Westman E, Aguilar C, Muehlboeck JS, Simmons A (2013) Regional magnetic resonance imaging measures for multivariate analysis in Alzheimer's disease and mild cognitive impairment. Brain Topogr 26:9-23. CrossRef Medline

Wisco JJ, Kuperberg G, Manoach D, Quinn BT, Busa E, Fischl B, Heckers S, Sorensen AG (2007) Abnormal cortical folding patterns within Broca's area in schizophrenia: evidence from structural MRI. Schizophr Res 94: 317-327. CrossRef Medline

Wolz R, Julkunen V, Koikkalainen J, Niskanen E, Zhang DP, Rueckert D, Soininen H, Lötjönen J (2011) Multi-method analysis of MRI images in early diagnostics of Alzheimer's disease. PLoS One 6:e25446. CrossRef Medline

Yu Y, Shen H, Zhang H, Zeng LL, Xue Z, Hu D (2013) Functional connectivity-based signatures of schizophrenia revealed by multiclass pattern analysis of resting-state fMRI from schizophrenic patients and their healthy siblings. Biomed Eng Online 12:10. CrossRef Medline

Zhang D, Wang Y, Zhou L, Yuan H, Shen D (2011) Multimodal classification of Alzheimer's disease and mild cognitive impairment. Neuroimage 55:856-867. CrossRef Medline

Zhou B, Nakatani E, Teramukai S, Nagai Y, Fukushima M (2012) Risk classification in mild cognitive impairment patients for developing Alzheimer's disease. J Alzheimers Dis 30:367-375. CrossRef Medline 